\title{
Impact of a Formal Patient Safety and Quality Improvement Curriculum: A Prospective, Controlled Trial
}

\author{
Nausheen Jamal, MD; Sarah N. Bowe, MD; Michael J. Brenner, MD; \\ Karthik Balakrishnan, MD, MPH; John P. Bent, MD
}

\begin{abstract}
Objective: To assess the impact of implementing a dedicated Patient Safety and Quality Improvement (PSQI) curriculum for otolaryngology residents.

Methods: Residents in two otolaryngology residency programs were recruited to participate in the study. Residents at institution A (intervention group) participated in a formal, newly developed, year-long PSQI curriculum. Residents at institution B (control group) participated in traditional, morbidity, and mortality conference-based PSQI education, with no formal curriculum in place. Curriculum participants completed anonymous surveys to assess learner satisfaction. Validated instruments were administered to assess for changes in resident confidence in the ability to develop PSQI projects, their attitudes toward patient safety, and PSQI-related knowledge. The number and quality of PSQI-related resident projects were also assessed.

Results: Survey responses demonstrated excellent learner satisfaction with the curriculum. Based on validated instrument-based responses, both programs demonstrated similar confidence scores $(P=0.05)$, safety attitudes $(P=0.82)$, and PSQI knowledge $(P=0.29)$ at the beginning of the year. The residents of institution A demonstrated significant improvement in confidence $(P=0.00009)$ and knowledge $(P=0.0006)$ after completing the curriculum, with no improvement noted for residents at institution $B$ in either confidence $(P=0.06)$ or knowledge $(P=0.79)$. Neither program demonstrated improvement in attitudes toward patient safety at the end of the year-long curriculum.

Conclusion: Implementing a formal curriculum dedicated to PSQI led to an improvement in PSQI-related project development confidence and PSQI knowledge. Attitudes toward safety did not improve over the course of a year. Longer-term studies involving multiple institutions and other interventions are needed to evaluate the impact and duration of changes that occur.

Key Words: Patient safety, quality improvement, practice-based learning and improvement, systems-based practice, otolaryngology.

Level of Evidence: $1 \mathrm{~b}$
\end{abstract}

Laryngoscope, 129:1100-1106, 2019

\section{INTRODUCTION}

In its landmark report To Err Is Human, the Institute of Medicine reported that up to 98 thousand people die in the United States each year due to preventable health care-related causes. ${ }^{1}$ As a result, the Accreditation Council for Graduate Medical Education (ACGME) recently updated postgraduate training by incorporating

From the Department of Otolaryngology-Head and Neck Surgery, Lewis Katz School of Medicine at Temple University (N.J.), Philadelphia, Pennsylvania; the Department of Otolaryngology-Head and Neck Surgery, San Antonio Uniformed Services Health Education Consortium (SAUSHEC) (s.N.B.), Ft. Sam Houston, TX; the Department of Otolaryngology-Head and Neck Surgery, University of Michigan School of Medicine (м.J.в.), Ann Arbor, Michigan; the Mayo Clinic Children's Center and Department of Otorhinolaryngology, Mayo Clinic (к.в.), Rochester, Minnesota; and the Department of Otorhinolaryngology-Head and Neck Surgery, Albert Einstein College of Medicine at Montefiore Medical Center (J.P.B.), Bronx, New York, U.S.A.

Additional supporting information may be found in the online version of this article.

Editor's Note: This Manuscript was accepted for publication on July $31,2018$.

The authors have no funding, financial relationships, or conflicts of interest to disclose.

Send correspondence to Nausheen Jamal, MD, Division of Otolaryngology, University of Texas Rio Grande Valley School of Medicine, 1210 W. Schunior St., EMEBL 3.145, Edinburg, TX 78541. E-mail: nausheen. jamal@utrgv.edu

DOI: 10.1002/lary.27527 patient safety and quality improvement (PSQI) requirements into its core graduation competencies. ${ }^{2}$ Furthermore, as part of the Next Accreditation System, graduate medical education (GME)-sponsoring institutions are required to undergo Clinical Learning Environment Review site visits, which primarily aim to determine whether these institutions' learning environments provide training in safe, high-quality patient care. ${ }^{3}$ This training now impacts professional practice in a practical way; the American Board of Otolaryngology now incorporates PSQI-reporting requirements into its Maintenance of Certification processes. ${ }^{4}$

Since 2000, a growing body of literature has investigated PSQI education in GME ${ }^{5-14}$ with notable progress in surgical disciplines in recent years. ${ }^{15-19}$ Nonetheless, PSQI education rarely appeared in otolaryngology literature until 2016. We therefore developed and implemented a formal PSQI curriculum for otolaryngology residents and compared outcomes against a matched control program using validated instruments. We predicted that residents participating in the PSQI curriculum would report improved confidence in developing PSQI projects, improved attitudes toward patient safety, and greater PSQI knowledge relative to residents not exposed to a formal curriculum. . $^{9,18,20,21}$ 


\section{MATERIALS AND METHODS}

This project was exempted by the institutional review boards of the two sponsoring institutions, Temple University Hospital (institution A, intervention program) and Montefiore Medical Center (institution B, control program).

\section{Curriculum Development}

Prior to implementation of the PSQI curriculum, institution A based its PSQI education on morbidity and mortality (M\&M) conference discussions, with no didactic or experiential curriculum. The formal PSQI curriculum was based on the Institute for Healthcare Improvement's (IHI) online module series, an expansion of their Open School Basic Certificate curriculum, as detailed in the online supplement (Appendix I and II). No formal PSQI curriculum was in place at institution B; resident education was based solely on regularly scheduled M\&M conferences.

For the experiential component of the curriculum, residents at institution A were expected to develop projects related to safety and quality and to submit a formal proposal for each project. Residents at institution $B$ were not specifically instructed to develop PSQI projects, but their program director requested proposals from any residents who worked on a PSQI-related project, whether for research or for quality improvement purposes.

\section{Assessment Tools}

Assessment of the PSQI curriculum was based on the Kirkpatrick model, which consists of four evaluation levels: 1) reaction, 2) learning, 3) behavior, and 4) results. ${ }^{22}$ Assessment of PSQI curricula may identify changes in confidence (Kirkpatrick level 2A) and attitudes toward safety (level 2A), measurable changes in knowledge (level 2B), and development and implementation of projects (level 4). Objective assessment of behavior change (level 3) is difficult but relies on observed behavioral adjustments such as increased "near-miss" episodes identified by residents. The Quality Improvement Confidence Instrument (QICI); Safety Attitudes Questionnaire (SAQ); Quality Improvement Knowledge Assessment Tool-Revised (QIKAT-R); and Quality Improvement Proposal Assessment Tool-7 (QIPAT-7) were selected as validated instruments measuring resident confidence, safety attitudes, knowledge, and PSQI project know-how. ${ }^{23-27}$ Learner reaction to the curriculum (Kirkpatrick level 1) was assessed using anonymous, Likert scale-based online surveys assessing resident opinions of each assigned web-based module, distributed via the Survey Monkey platform. An online supplement details these assessment methods (Appendix III).

\section{Study Protocol}

At institution A, residents from all five postgraduate year (PGY) levels enrolled in the Patient Safety and Quality Improvement curriculum during one academic year. Survey responses were compared to those of residents from institution $\mathrm{B}$, which cares for a similar patient population (urban, academic tertiary referral center, primarily Medicaid-based). Institution B residents did not participate in a formal PSQI curriculum beyond standard departmental M\&M conference.

Pre- and postcurriculum assessments (QICI, SAQ, and QIKAT-R) were administered to residents and faculty of both groups according to the protocol depicted in Figure 1. Residents completed all three instruments at both time points, whereas faculty completed only the SAQ. Faculty were included in order to assess the safety culture for the department as a whole (beyond the residency program). Completed QIKAT-R instruments and PSQI project proposals were graded by three reviewers (s.N.B., M.J.

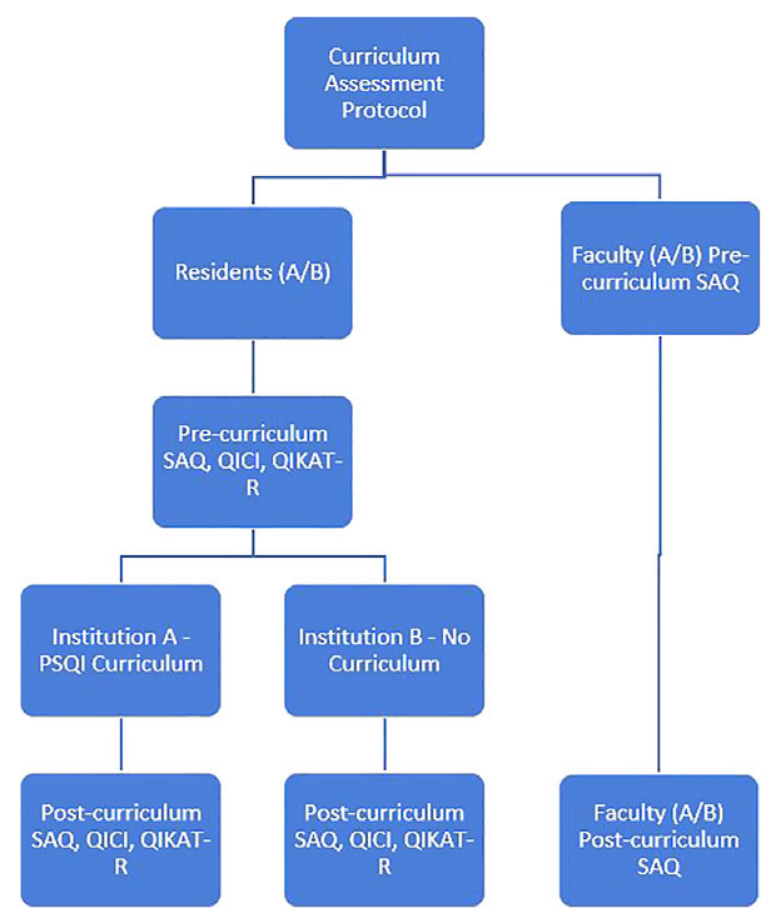

Fig. 1. Study protocol. $A=$ institution $A$ (intervention group); $B=$ institution B (control group); PSQI = Patient Safety and Quality Improvement; $\mathrm{QICI}=$ Quality Improvement Confidence Instrument; QIKAT$\mathrm{R}=$ Quality Improvement Knowledge Assessment Tool-Revised; $S A Q=$ Safety Attitudes Questionnaire. [Color figure can be viewed in the online issue, which is available at www.laryngoscope.com.]

в., к.в.) who were blinded both to the institution and to whether the form was completed at the beginning or end of the academic year. The QICI and SAQ were collected without identifying information, except for notation of time point and institution. The SAQ asked individuals to identify as a resident or faculty physician.

\section{Statistical Analysis}

Results of the QICI, SAQ, and QIKAT-R were compared between pre- and postcurriculum implementation and across institutions A and B using two-tailed Student $t$ tests, with $P$ values $<0.05$ considered significant. Paired $t$ tests were used to compare mean pre- and postcurriculum scores for each institution, and equal variance was assumed in these comparisons. The same analysis was performed to compare postcurriculum QIKAT-R scores between institutions. Individual results were not tested against one another due to anonymized survey collection and because not all residents completed postintervention questionnaires, thus making analysis for causation over time not possible. QIPAT-7 and postmodule completion online survey results were analyzed using descriptive statistics.

\section{RESULTS}

QICI, SAQ, and QIKAT-R completion rates for residents $(n=11)$ in the intervention group (institution A) were $100 \%$ for each instrument, both before and after curriculum implementation. Faculty SAQ completion rates at the same institution $(\mathrm{n}=12)$ were $83 \%$ before and $75 \%$ after curriculum implementation. Instrument completion rates for the institution $B$ residents $(n=20)$ were $90 \%$ for each instrument at the beginning of the academic year 
Resident Assessment of Web-based Modules

$60.0 \%$

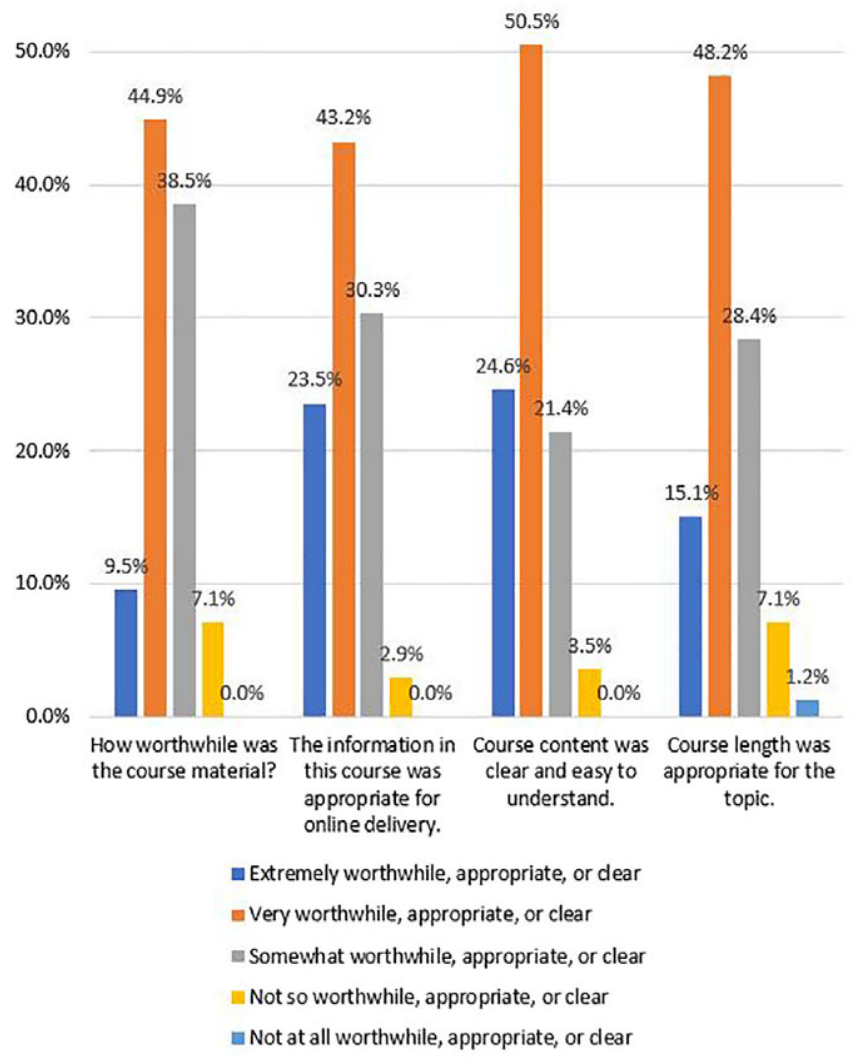

Fig. 2. Resident assessment of web-based modules. Each module was based on a Likert-based scale. The majority of residents felt that the modules were very or extremely worthwhile, appropriate, and/or clear. [Color figure can be viewed in the online issue, which is available at www.laryngoscope.com.] and $50 \%$ at year-end. Faculty SAQ completion rates at institution $B(n=20)$ were $60 \%$ at the beginning of the year and $65 \%$ at year-end.

\section{Learner Reaction to Curriculum (Kirkpatrick Level 1)}

Web-based module completion rate for institution $\mathrm{A}$ residents was $100 \%$ for all modules. Following each classroom session module, evaluation questionnaires were anonymously distributed to institution A residents via Survey Monkey. The mean survey completion rate for all 17 evaluation questionnaires was $62 \%$, with a range of $27 \%$ to $100 \%$ (mode $82 \%$ ). Figure 2 displays results for all 17 modules. The majority of residents responded that modules were "very" or "extremely" worthwhile $(67 \%)$, appropriate for online delivery (54\%), clear and easy to understand (75\%), and of appropriate length (63\%).

\section{Changes in Confidence (Kirkpatrick Level 2A)}

The QICI evaluates resident confidence in the six steps of PSQI project development and implementation. Figure 3 and Table I show mean responses ranging from 1 ("not at all confident") to 5 ("very confident") for each program at each step, as well as overall confidence scores (average of all six steps).

Institution A's mean overall precurriculum confidence score indicated overall neutral to low confidence (2.93 [95\% confidence interval $(\mathrm{CI})=2.76-3.11])$. Institution B's mean overall precurriculum confidence levels suggested slightly better than neutral confidence (3.32 [95\% CI $=3.20-3.44]$ ).

By the end of the curriculum, institution A mean overall confidence levels increased significantly to 3.94 $(95 \%$ CI $=3.86-4.03, P=0.00009)$. In addition, each

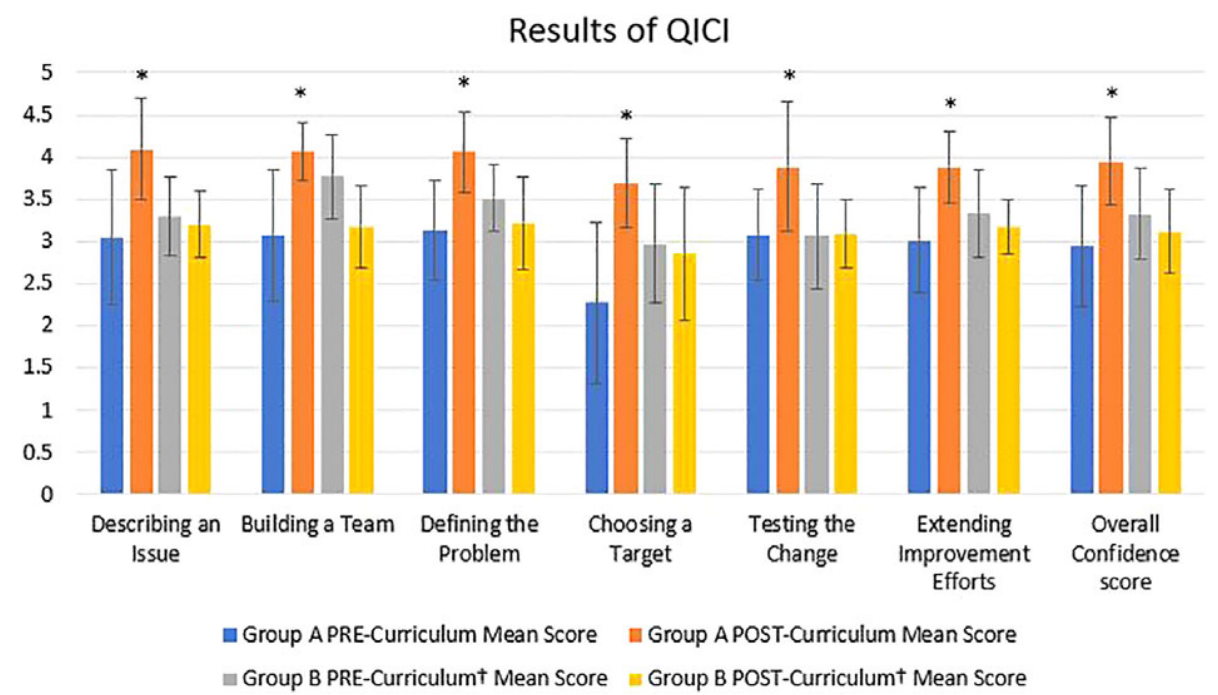

Fig. 3. Results of the QICI. Mean pre- and postcurriculum scores for each step of Patient Safety and Quality Improvement project development (as described by the QICI) are shown for both institutions. Scores are based on a Likert scale for which 1 indicates "not at all confident"; 3 indicates "neutral"; and 5 indicates "very confident." Postcurriculum scores were significantly improved for each step in the intervention group (institution A) but unchanged for the control group (institution B). Error bars indicate standard deviation; asterisk (*) indicates $P<0.05$. ${ }^{\dagger}$ Institution A's curriculum. Institution B's residents have no formal PSQI curriculum in place and are serving as controls. PSQI = Patient Safety and Quality Improvement; $\mathrm{QICl}=$ Quality Improvement Confidence Instrument. [Color figure can be viewed in the online issue, which is available at www.laryngoscope.com.] 
TABLE I.

Results of the Quality Improvement Confidence Instrument

\begin{tabular}{|c|c|c|c|c|}
\hline $\begin{array}{l}\text { QICI Results: Steps } \\
\text { of PSQI Project } \\
\text { Development }\end{array}$ & $\begin{array}{c}\text { Group A } \\
\text { Pre-/ } \\
\text { Post-Change }\end{array}$ & $P$ Value & $\begin{array}{c}\text { Group B } \\
\text { Pre-/ } \\
\text { Post-Change }\end{array}$ & $\begin{array}{c}P \\
\text { Value }\end{array}$ \\
\hline Describing an issue & 1.05 & 0.002 & -0.09 & 0.605 \\
\hline Building a team & 1.00 & 0.001 & -0.59 & 0.493 \\
\hline Defining the problem & 0.93 & 0.002 & -0.29 & 0.917 \\
\hline Choosing a target & 1.41 & 0.002 & -0.12 & 0.868 \\
\hline Testing the change & 0.81 & 0.023 & 0.02 & 0.932 \\
\hline $\begin{array}{l}\text { Extending } \\
\text { improvement } \\
\text { efforts }\end{array}$ & 0.87 & 0.007 & -0.17 & 0.714 \\
\hline $\begin{array}{l}\text { Overall confidence } \\
\text { score }\end{array}$ & 1.01 & 0.00009 & -0.21 & 0.065 \\
\hline
\end{tabular}

Mean pre- and postcurriculum scores for each step of Patient Safety and Quality Improvement (PSQI) project development (as described by the $\mathrm{QICl)}$ are shown for both institutions. Scores are based on a Likert scale: 1 indicates "not at all confident"; 3 indicates "neutral"; and 5 indicates "very confident." Postcurriculum scores were significantly improved for each step in the intervention group (institution A) but unchanged for the control group (institution B).

PSQI = Patient Safety and Quality Improvement; $\mathrm{QICI}=$ Quality Improvement Confidence Instrument.

individual subscale improved significantly. Institution B, however, had stable confidence levels, decreasing in one area. The mean overall confidence score (3.12 [95\% $\mathrm{CI}=3.04-3.19])$ showed no statistical difference compared to the mean precurriculum score $(3.32[P=0.065])$.

\section{Changes in Attitudes Toward Safety (Kirkpatrick Level 2A)}

The SAQ assesses seven safety climate subscales as well as the overall safety attitude climate from a respondent's viewpoint, with scores $\geq 75$ indicating a positive climate. Figure 4 (residents) and Figure 5 (faculty) list the SAQ results for both institutions' residents and faculty.

Institution A demonstrated an overall resident safety attitudes climate score of 68 prior to curriculum implementation. The baseline climate subscores were essentially similar to those seen for institution B $(P=0.82)$. The overall resident safety attitudes score and all subscale scores for institution A were unchanged for its residents' postcurriculum (all $P>0.05$ ). Similarly, overall safety climate was unchanged over the academic year for institution $\mathrm{B}$ residents $(P=0.71)$, with no significant changes in any subscale (all $P>0.05$ ).

Regarding faculty SAQ responses, for institution A the precurriculum overall climate responses averaged 59 . This showed no improvement at year end $(P=0.265)$. Only one subscale showed significant change; safety climate improved by 18.8 points $(P=0.02)$. At institution $\mathrm{B}$, the overall safety attitudes climate score and climate subscales for faculty were unchanged $(P>0.05$ for all).

\section{Changes in Knowledge (Kirkpatrick Level 2B)}

The QIKAT-R is designed to objectively assess knowledge of fundamental PSQI concepts. Responses from residents at both institutions were graded on a scale of 0 (worst possible score) to 27 (perfect score) (Fig. 6) (Supporting Table SI, available online). Prior to PSQI curriculum implementation, institution A's mean QIKAT$\mathrm{R}$ score (13.97 [95\% CI = 11.04-16.90]) was statistically no different from that of institution B (12.35 $[P=0.29$, 95\% CI $=7.02-17.68]$ ). After completing the curriculum, the mean QIKAT-R scores for institution A for all residents improved 8.23 points $(P=0.0006)$ to $22.15(95 \%$

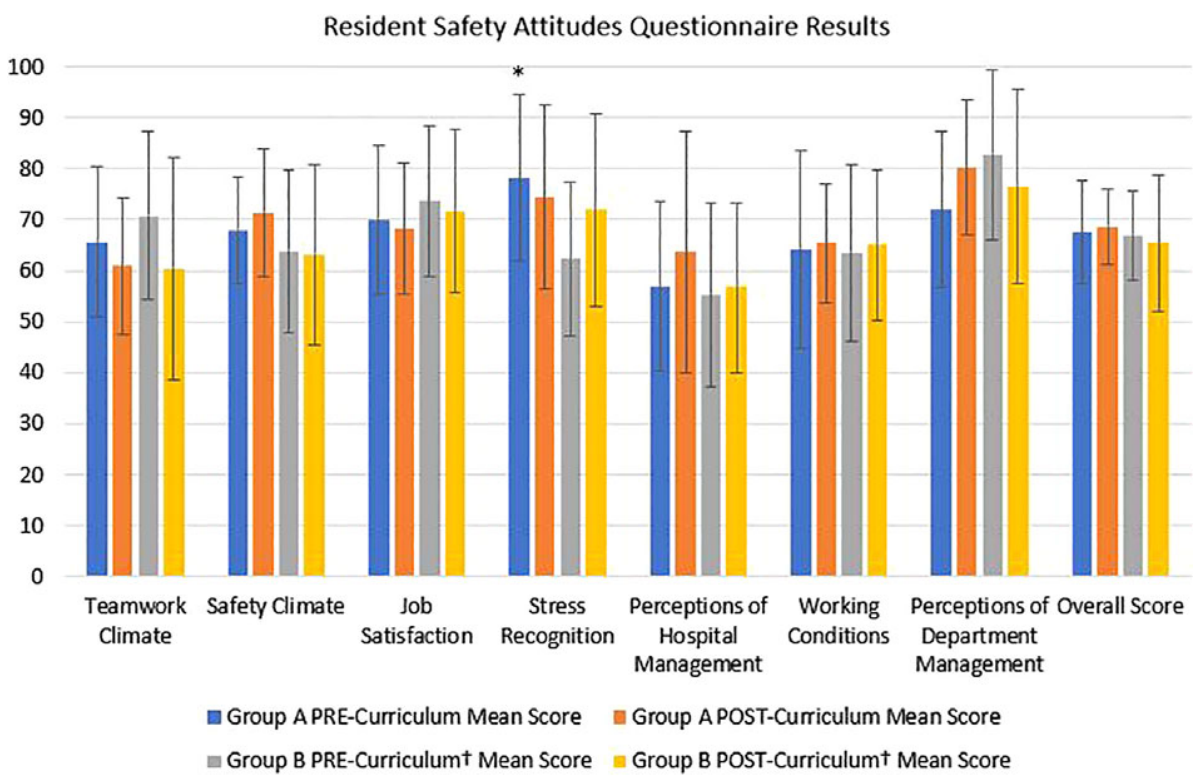

Fig. 4. Results of the Safety Attitudes Questionnaire for residents. A positive climate is indicated by a score of 75 or higher. Baselines scores were similar for both groups, with the exception of a higher stress recognition score for group $\mathrm{A}(P=0.015)$. No changes were noted by the end of the curriculum year in either group. Error bars indicate standard deviation; asterisk (*) indicates $P<0.05$. ${ }^{\dagger}$ Institution A's curriculum. Institution B's residents have no formal PSQI curriculum in place and are serving as controls. PSQI = Patient Safety and Quality Improvement. [Color figure can be viewed in the online issue, which is available at www.laryngoscope.com.] 


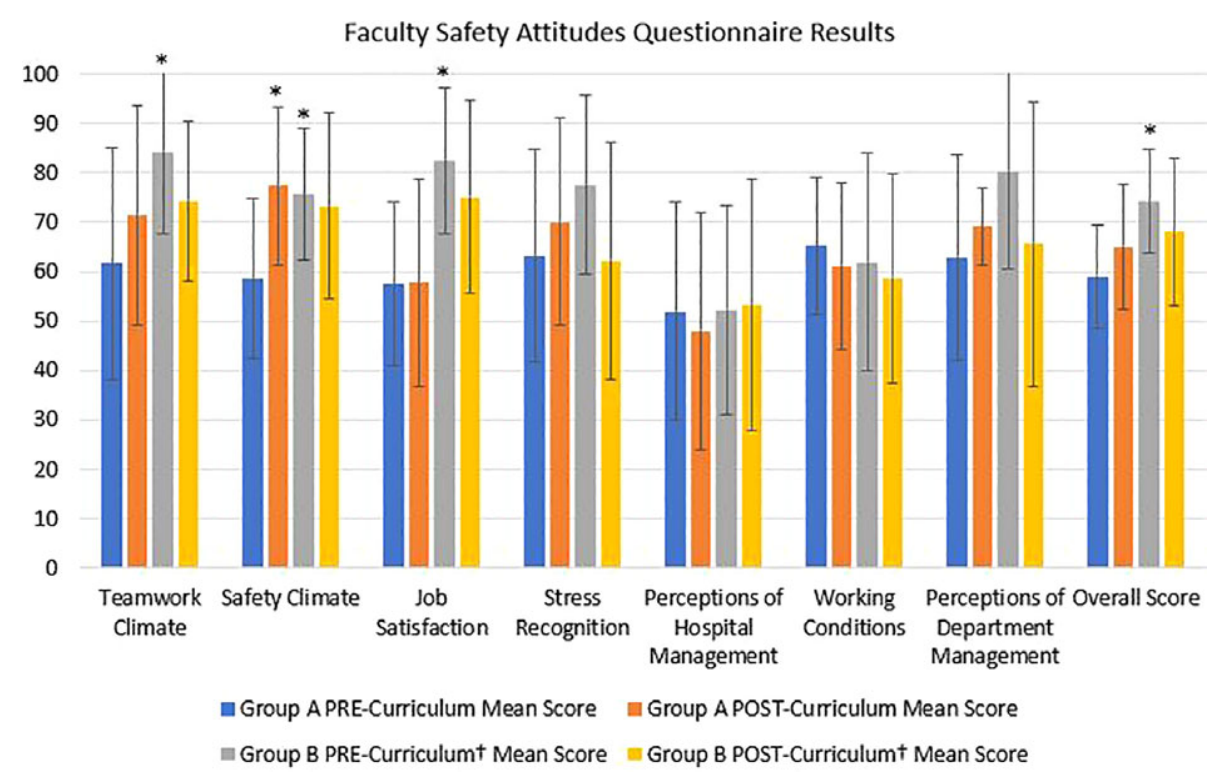

Fig. 5. Results of the Safety Attitudes Questionnaire for faculty. A positive climate is indicated by a score of 75 or higher. Baselines scores were similar for about half of the subscales for the two groups, whereas teamwork climate $(P=0.016)$, safety climate $(P=0.023)$, job satisfaction $(P=0.001)$, and overall score $(P=0.002)$ were all higher for institution $\mathrm{B}$. The only change noted by the end of the curriculum year was improvement in the safety climate subscale for group A $(P=0.021)$. Error bars indicate standard deviation; asterisk $(*)$ indicates $P<0.05$. 'Institution A's curriculum. Institution B's residents have no formal PSQI curriculum in place and are serving as controls. [Color figure can be viewed in the online issue, which is available at www.laryngoscope.com.]

$\mathrm{CI}=18.52-25.79)$. In contrast, institution B's residents mean precurriculum score was essentially unchanged (12.77, $[P=0.79,95 \% \mathrm{CI}=9.84-15.70])$. Therefore, postcurriculum scores were significantly higher at institution A when compared to institution B $(P=0.0000015)$.

\section{Project Development (Kirkpatrick Level 4)}

A total of four PSQI project proposals were submitted throughout the course of the curriculum as residents developed project ideas (Supporting Table SII, available online), and all four projects are currently in midimplementation phase. All proposals were developed by residents at institution A. Each was evaluated using the QIPAT-7 mechanism. On a scale of 7 (poorest possible quality) to 35 (highest possible quality), these proposals received scores ranging from 13 to 23. All proposal scores fell into the "meets expectations" category, and none were classified as "exceed expectations."

\section{DISCUSSION}

We investigated the impact of a year-long PSQI curriculum on residents' confidence, attitudes, and knowledge of PSQI relative to a matched control program. Kirkpatrick level 1 evaluation ("reaction") demonstrated a positive learner reaction to the web-based module component of the curriculum. Over half of institution A's residents reported the course material was "very" or "extremely" worthwhile. They similarly endorsed that the content was clear and easy to understand, and the information was appropriate for online delivery.

Kirkpatrick level 2A evaluation (“confidence") via the QICI showed initial neutral-to-low levels of confidence
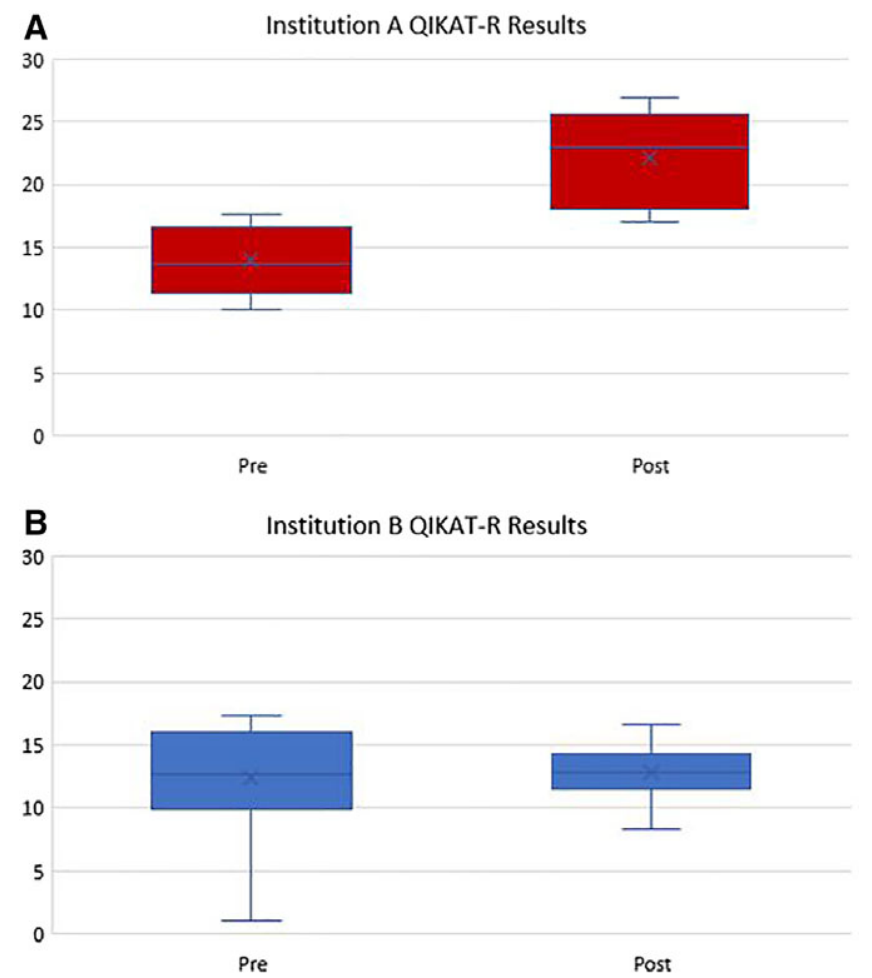

Fig. 6. Box-and-whisker plots of mean QIKAT-R scores for institution $A(A)$ and institution $B(B)$. Shaded area marks the middle two quartiles. The horizontal bar identifies the median, and " $X$ " marks the mean score for each group. Precurriculum scores for the two programs were similar $(P=0.24)$. Only the intervention group (institution A) showed a significant improvement in QIKAT-R score by the end of the curriculum (see Table SII). QIKAT-R = Quality Improvement Knowledge Assessment Tool-Revised. [Color figure can be viewed in the online issue, which is available at www.laryngoscope.com.] 
for PSQI project development for both resident groups. At year end, institution A's residents showed significantly improved confidence scores in all six project development steps. Scores increased from "neutral" to "confident" range for all steps, whereas institution B's residents remained "neutral." Kirkpatrick level 2A evaluation ("attitudes") via the SAQ demonstrated a lack of positive overall safety attitudes culture in both institutions among both residents and faculty. At year's end, this measure remained essentially unchanged, with the notable exception of perceived safety climate improvement as noted by the faculty of institution A. Thus, gains in PSQI knowledge are not necessarily accompanied by improvement in departmental attitudes, although increased resident interest and involvement in PSQI may impact faculty perceptions to some extent. Wong et al. also noted a minimal impact on learner attitudes within quality improvement education, although they found that this was likely related to many trainees having favorable attitudes toward PSQI at baseline. ${ }^{28}$ Although a 1-year curriculum may be insufficient to change entrenched attitudes, it is also possible that curricular innovation must be accompanied by commitment at the departmental and institutional leadership level to promote a safety-oriented culture. Furthermore, changing PSQI education is likely only a first step toward changing culture. By building knowledge and confidence, one can work toward changing culture. Cultural changes happen gradually and will require more than just an educational program to make any dramatic shifts. Continued assessment in future years will help track trends in safety attitudes. Despite underwhelming SAQ scores, there is reason for optimism. Several residents involved in the PSQI curriculum have since volunteered to join hospital-based safety and quality-improvement committees, suggesting that SAQ may not adequately capture engagement. These findings reflect that changes in culture are difficult to achieve and likely require both time and institutional prioritization.

Kirkpatrick level 2B evaluation ("knowledge") also demonstrated a positive change, with evidence of improvement in PSQI knowledge. Residents at institution A demonstrated significant improvement in their QIKAT$\mathrm{R}$ scores, whereas residents at institution $\mathrm{B}$ showed no statistically significant improvement.

Evaluation of resident PSQI project proposals provides data on Kirkpatrick level 4 ("results"). Every resident at institution A participated in a PSQI project, and all projects were conducted in groups. The four residentled projects developed during the course of the year were a dramatic shift from years prior when no PSQI projects were developed; the new curriculum likely accounted for this increase. In contrast, residents at institution B submitted no PSQI-related project proposals.

Now that education and experience in PSQI is mandated by the ACGME, otolaryngology faculty must determine how best to meet this requirement. A recent systematic review of PSQI education in otolaryngology demonstrated an increase in publications of PSQI projects related to resident education since $2008 .^{29}$ Interestingly, none of the articles in this series pertained to formally integrated curricula on the fundamentals of PSQI. In fact, only a small number of the articles focused on integrating PSQI otolaryngology resident educational curricula. Most of these were published after the time frame of the systematic review., ${ }^{4,21,30-33}$

A primary example of a PSQI curriculum publication is that of Bowe et al., who also used the IHI web-based module series (restricted to the IHI Open School Basic Certificate program). The study involved PGY2, PGY3, and PGY4 otolaryngology residents and supplemented the IHI program with faculty-led lectures to reinforce module content. ${ }^{21}$ Residents served as their own controls. Learners were then assessed using several survey instruments, including two attitude-based surveys for PGY2s and the QIKAT-R and QICI for PGY3s and PGY4s. Similar to the present study, they found significant improvement in the QIKAT-R scores but no significant change in attitude-based surveys or the QICI results, which is in contrast to the current study.

In a commentary from 2015, McCormick et al. suggest beginning resident PSQI curricula with formal didactic lectures to introduce fundamental PSQI concepts. ${ }^{4}$ These didactics can be supplemented with web-based modules, faculty-led workshops, and "off-the-cuff" real-time safety and quality bedside discussions with residents. Experiential learning can be added by enhancing M\&M to include systems-based discussions, root cause analyses of complications and near misses, and development of resident-driven projects. The authors acknowledged potential challenges to implementing these interventions, including lack of faculty knowledge and interest, lack of resident time and interest, and curriculum issues.

Publications evaluating otolaryngology PSQI curricula have been limited when compared with other specialties, perhaps because such curricula are not yet established in most residency programs. In a survey of program directors, Bowe found that although $90 \%$ of respondents felt PSQI education is important for a resident's future success in otolaryngology, only $23 \%$ had a formal curriculum in their program. ${ }^{34}$ The greatest barriers to PSQI curriculum integration cited by program directors were lack of faculty PSQI expertise (cited by $75 \%$ ) and lack of time due to competing educational demands (cited by 90\%)—both predicted by McCormick et $a{ }^{4}{ }^{4}$ Fortunately, with numerous educational resources available, faculty content expertise is no longer necessary a priori in order for residents to learn PSQI concepts. ${ }^{6,32}$

Another challenge after implementing a PSQI curriculum is evaluation of its success. Otolaryngology residency programs are much smaller than their counterparts in other fields, making it difficult to measure impact and external validity of single program interventions. One must thus rely on using two (or more) different programs to assess the potential influence of a PSQI curriculum. Certainly, involvement of more than one program results in unavoidable heterogeneity among study participants. For example, one cannot control for factors such as individual resident similarities or institutional outcomes. However, the two programs chosen to participate in this study were selected due to their numerous similarities. Both are academic institutions that serve primarily Medicaid populations; both care for similar high volume, urban poor populations who face 
similar social determinants of care; baseline knowledge and attitude survey scores were similar between the two institutions; and both had the same preintervention PSQI education program in place for their residents (M\&M conferences based on resident-reported complications). Studies involving multiple programs are difficult to coordinate as a result of heterogeneity in structure and culture. This consideration was relevant to the present study. Implementing a new curriculum across multiple residency programs, along with rigorous evaluation methods, is a very resource-intensive endeavor. This is related to one of the limitations of this study, namely the low completion rates of survey instruments, particularly in institution B.

Both the study design (use of two nonidentical programs) and the lack of difference in patient safety attitudes after the educational intervention limit the conclusions that can be drawn from this study. Nonetheless, this study provides evidence that implementing a formal didactic and experiential curriculum in PSQI can improve resident knowledge and confidence. Changes in attitudes and safety culture are more elusive and likely take time, creativity, and championing by leadership. Additionally, this study shows that without a formal, dedicated PSQI curriculum, residents are unlikely to build the confidence and knowledge base necessary to be actively involved in performance improvement during residency and beyond.

\section{CONCLUSION}

Beyond meeting ACGME mandates, implementation of a PSQI curriculum is an effective way to develop residents' knowledge of safety and quality concepts. A dedicated curriculum also builds confidence, both which are necessary skills for all future physicians in order to participate PSQI efforts throughout their careers. Longer-term studies involving multiple institutions and additional educational interventions will be helpful in assessing the nature and durability of changes that occur, as well as tracking the impact upon safety attitudes and culture.

\section{Acknowledgment}

The authors are grateful to John H. Krouse, MD, $\mathrm{PhD}, \mathrm{MBA}$; and Brian Nussenbaum, MD, for their support and mentorship of this project.

This article was originally prepared as a Triological Society Candidate thesis for the first author (N.J.).

\section{BIBLIOGRAPHY}

1. Kohn LT, Corrigan J, Donaldson M, ed. To Err is Human: Building a Safer Health System. Washington, DC: National Academy Press; 1999.

2. Accreditation Council for Graduate Medical Education. ACGME program requirements for graduate medical education in otolaryngology. Published July 1, 2014. Available at: https://www.acgme.org/Portals/0/280 otolaryngology_PRs_RC.pdf. Accessed July 28, 2017.

3. Clinical Learning Environment Review Committee. CLER Pathways to Excellence: Expectations for an optimal clinical learning environment to achieve safe and high-quality patient care. Published 2014. Available at: https://www.acgme.org/Portals/0/PDFs/CLER/CLER_ Brochure.pdf. Accessed July 28, 2017.

4. McCormick ME, Stadler ME, Shah RK. Embedding quality and safety in otolaryngology-head and neck surgery education. Otolaryngol Head Neck Surg 2015;152:778-782.
5. Oyler J, Vinci L, Arora V, Johnson J. Teaching internal medicine residents quality improvement techniques using the ABIM's practice improvement modules. J Gen Intern Med 2008;23:927-930.

6. Peters AS, Kimura J, Ladden MD, March E, Moore GT. A self-instructional model to teach systems-based practice and practice-based learning and improvement. J Gen Intern Med 2008;23:931-936.

7. Vinci LM, Oyler J, Johnson JK, Arora VM. Effect of a quality improvement curriculum on resident knowledge and skills in improvement. Qual Saf Health Care 2010;19:351-354.

8. Tentler A, Feurdean M, Keller S, Kothari N. Integrating a resident-driven longitudinal quality improvement curriculum within an ambulatory block schedule. J Grad Med Educ 2016;8:405-409.

9. Potts S, Shields S, Upshur C. Preparing future leaders: an integrated quality improvement residency curriculum. Fam Med 2016;48:477-481.

10. Philibert I, Gonzalez Del Rey JA, Lannon C, Lieh-Lai M, Weiss KB. Quality improvement skills for pediatric residents: from lecture to implementation and sustainability. Acad Pediatr 2014;14:40-46.

11. Gupta M, Ringer S, Tess A, Hansen A, Zupancic J. Developing a quality and safety curriculum for fellows: lessons learned from a neonatology fellowship program. Acad Pediatr 2014;14:47-53.

12. Posner KL, Kendall-Gallagher D, Wright IH, Glosten B, Gild WM Cheney FW Jr. Linking process and outcome of care in a continuous quality improvement program for anesthesia services. Am J Med Qual 1994;9: 129-137.

13. Pronovost PJ, Kazandjian VA. A new learning environment: combining clinical research with quality improvement. J Eval CLin Pract 1999;5:33-40.

14. Mayer TA. Industrial models of continuous quality improvement. Implications for emergency medicine. Emerg Med Clin North Am 1992;10: $523-547$.

15. Sellers MM, Hanson K, Schuller M, et al. Development and participant assessment of a practical quality improvement educational initiative for surgical residents. J Am Coll Surg 2013;216:1207-1213, 1213.e1.

16. O'Heron CT, Jarman BT. A strategic approach to quality improvement and patient safety education and resident integration in a general surgery residency. J Surg Educ 2014;71:18-20.

17. Dougherty PJ, Kromrei H. CORR curriculum-orthopaedic education: quality improvement in resident education. Clin Orthop Relat Res 2016;474: 1939-1942.

18. Mrdutt MM, Isbell CL, Regner JL, et al. NSQIP-based quality improvement curriculum for surgical residents. J Am Coll Surg 2017;224:868-874.

19. Sepulveda D, Varaklis K. Implementing a multifaceted qualityimprovement curriculum in an obstetrics-gynecology resident continuityclinic setting: a 4-year experience. J Grad Med Educ 2012;4:237-241.

20. Ogrinc G, Headrick LA, Morrison LJ, Foster T. Teaching and assessing resident competence in practice-based learning and improvement. J Gen Intern Med 2004;19:496-500.

21. Bowe SN, Laury AM, Kepchar JJ, Lospinoso J. Programmatic assessment of a comprehensive quality improvement curriculum in an otolaryngology residency. Otolaryngol Head Neck Surg 2016;155:729-732.

22. Kirkpatrick JD, Kirkpatrick WK. Kirkpatrick's Four Levels of Training Evaluation. East Peoria, IL: ATD Press; 2016.

23. Hess BJ, Johnston MM, Lynn LA, Conforti LN, Holmboe ES. Development of an instrument to evaluate residents' confidence in quality improvement. Jt Comm J Qual Saf 2013;39:502-510.

24. Sexton JB, Helmreich RL, Neilands TB, et al. The safety attitudes questionnaire: psychometric properties, benchmarking data, and emerging research. BMC Health Serv Res 2006;6:44.

25. Singh MK, Ogrinc G, Cox KR, et al. The quality improvement knowledge application tool revised (QIKAT-R). Acad Med 2014;89:1386-1391.

26. Morrison LJ, Headrick LA, Ogrinc G, Foster T. The quality improvement knowledge application tool: an instrument to assess knowledge application in practice-based learning and improvement. J Gen Intern Med 2003; 18:S250.

27. Leenstra JL, Beckman TJ, Reed DA, et al. Validation of a method for assessing resident physicians' quality improvement proposals. J Gen Intern Med 2007;22:1330-1334.

28. Wong BM, Levinson W, Shojania KG. Quality improvement in medical education: current state and future directions. Med Ed 2012;46:107-119.

29. Gettelfinger JD, Baulk PB, Schmalbach CE. Patient safety and quality improvement in otolaryngology education: a systematic review. Otolaryngol Head Neck Surg 2017;156:991-998.

30. Laury AM, Bowe SN, Lospinoso J. Integrating morbidity and mortality core competencies and quality improvement in otolaryngology. JAMA Otolaryngol Head Neck Surg 2017;143:135-140.

31. Spielman DB, Hsueh WD, Choi KY, Bent JP. From morbidity and mortality to quality improvement: effects of a structured and interactive otolaryngology conference. OTO Open. doi: 10.1177/2473974X17692775.

32. Jamal N. Patient safety and quality improvement education in otolaryngology residency: Preliminary look at a module-based approach. OTO Open 2017. doi: 10.1177/2473974X17698647.

33. Morrison RJ, Bowe SN, Brenner MJ. Teaching quality improvement and patient safety in residency education: strategies for meaningful resident quality and safety initiatives. JAMA Otolaryngol Head Neck Surg 2017; 143:1069-1070.

34. Bowe SN. Quality improvement in otolaryngology residency: survey of program directors. Otolaryngol Head Neck Surg 2016;154:349-354. 